\title{
Osteomyelitis of Bilateral Femoral Heads After Childbirth: A Case Report
}

\author{
Kyung Soo Lee, $\mathrm{MD}^{1}$, Sangwon Kong, $\mathrm{MD}^{1}$, Junho Kim, $\mathrm{MD}^{1}$, Taikon Kim, $\mathrm{MD}, \mathrm{PhD}^{1}$, \\ Chan Beom Choi, MD, PhD², Yee-Suk Kim, MD, PhD ${ }^{3}$, Kyu Hoon Lee, MD, PhD ${ }^{1}$ \\ Departments of ${ }^{1}$ Rehabilitation Medicine, ${ }^{2}$ Reumatology, and ${ }^{3}$ Orthopedic Surgery, Hanyang University Medical Center, \\ Hanyang University College of Medicine, Seoul, Korea
}

Hip and pelvic pain during pregnancy or after delivery is a common problem in young females, and in most cases this problem has a self-limiting course. The patient described in this case suffered from severe hip pain after childbirth. MR imaging study was performed and it showed arthritis of bilateral hip joints and osteomyelitis of femoral heads with an abscess in the surrounding muscle. Infection, such as septic arthritis or osteomyelitis, is an extremely rare cause of peripartum joint pain. The patient's clinical symptoms and laboratory findings improved with antibiotic therapy. However, limitation of motion of the bilateral hip joints persisted although the patient continued rehabilitative therapy for 15 months, and the patient had to undergo bilateral total hip replacement. Hereby, we present a case of severe osteomyelitis and pyogenic arthritis of bilateral femoral heads and hip joints after delivery, which eventually required bilateral total hip replacement.

Keywords Osteomyelitis, Pregnancy, Total hip replacement

\section{INTRODUCTION}

Pelvic region pain, such as hip, pelvis, or lower back pain, is commonly observed during pregnancy [1]. In a previous study, in most cases, the pain was considered to occur due to heavy workload, joint laxity, and hormonal changes during pregnancy and peripartum period [2]. The pain often disappears soon after delivery. However,

Received June 19, 2014; Accepted September 2, 2014

Corresponding author: Kyu Hoon Lee

Department of Rehabilitation Medicine, Hanyang University Medical Center, Hanyang University College of Medicine, 222 Wangsimni-ro, Seongdong-gu, Seoul 133-791, Korea

Tel: +82-2-2290-9354, Fax: +82-2-2282-0772, E-mail: dumitru1@hanyang. ac.kr

(c) This is an open-access article distributed under the terms of the Creative Commons Attribution Non-Commercial License (http://creativecommons. org/licenses/by-nc/4.0) which permits unrestricted noncommercial use, distribution, and reproduction in any medium, provided the original work is properly cited.

Copyright $\odot 2015$ by Korean Academy of Rehabilitation Medicine in about $10 \%-15 \%$ of the cases, the pain persists for more than three months after delivery [3]. Although it is not common, infection, such as septic arthritis or osteomyelitis, could be a cause of chronic joint pain after delivery. Gamble et al. [4] reported a case of osteomyelitis of the pubic symphysis after childbirth.

This type of infection around the joints or bones can occur due to many reasons. For example, hematogenous seeding of bacteria is the most common cause of septic joints. Joint aspiration or local corticosteroid joint injection may also cause septic arthritis. In addition, joint trauma without a break in the skin or penetrating trauma could be the cause of septic arthritis [5].

Recently, we encountered a patient suffering from severe hip joint pain after delivery. We discovered that the pain was caused due to severe osteomyelitis and pyogenic arthritis of bilateral femoral heads and hip joints after a normal spontaneous vaginal delivery (NSVD). The pa- 
tient eventually required bilateral total hip replacement because of severe joint pain and impairment in daily activities.

We could not find any literature that described bilateral septic hip joints and osteomyelitis of femoral heads after a NSVD, which eventually needed surgical treatment because of irreversible damage to the joints. Thereby, we present our experience in the form of a case report.

\section{CASE REPORT}

A 35-year-old female gave birth to a preterm baby through a NSVD. She had no medical history other than that of one abortion because of ectopic pregnancy two years before delivery. She started complaining of pain in the bilateral hip area 12 days after delivery. High fever $\left(38.4^{\circ} \mathrm{C}\right)$ with tenderness and heating sensation on the bilateral hip area also developed 17 days after delivery.
The blood tests showed leukocytosis $\left(14,250 / \mathrm{mm}^{3}\right)$ and increased C-reactive protein (CRP) level of $15.2 \mathrm{mg} / \mathrm{dL}$. The physicians decided to obtain magnetic resonance (MR) images of the sacroiliac joints. The MR images demonstrated osteomyelitis of bilateral femoral heads, left sacroiliitis, and a suspicious abscess in the left iliacus muscle (Fig. 1).

Antibiotic therapy with cefminox was started immediately and it was changed to cefmenoxime one week later because of sustained fever. The antibiotic therapy was continued for 5 weeks, and her body temperature returned to normal and laboratory results showed evidence of mild degree of inflammation. However, severe leukocytopenia due to antibiotic therapy was discovered and the patient was transferred to our institution for further management. MR images were re-obtained immediately, and they still showed osteomyelitis with pyogenic arthritis and surrounding myositis of bilateral hip joints,
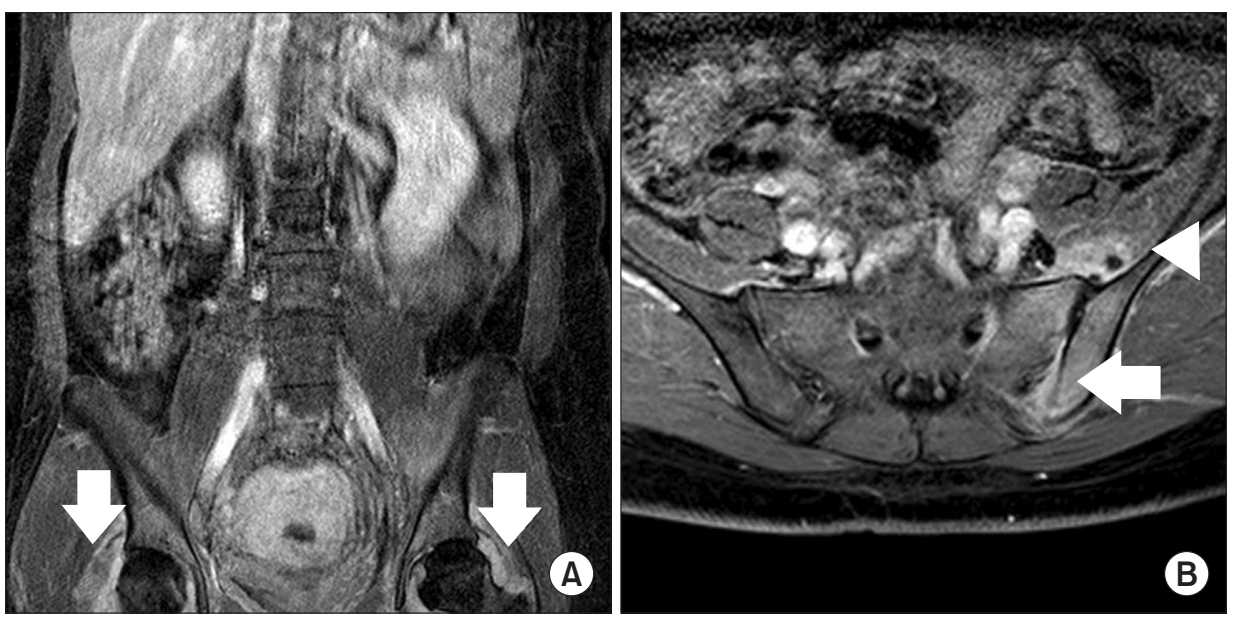

Fig. 1. Magnetic resonance images of the sacroiliac joint. Coronal enhanced T1-weighted image shows osteomyelitis of bilateral femoral heads (A), and transverse enhanced T1-weighted image showed left sacroiliitis (arrow), and a suspicious abscess in the left iliacus muscle (arrow head) (B).
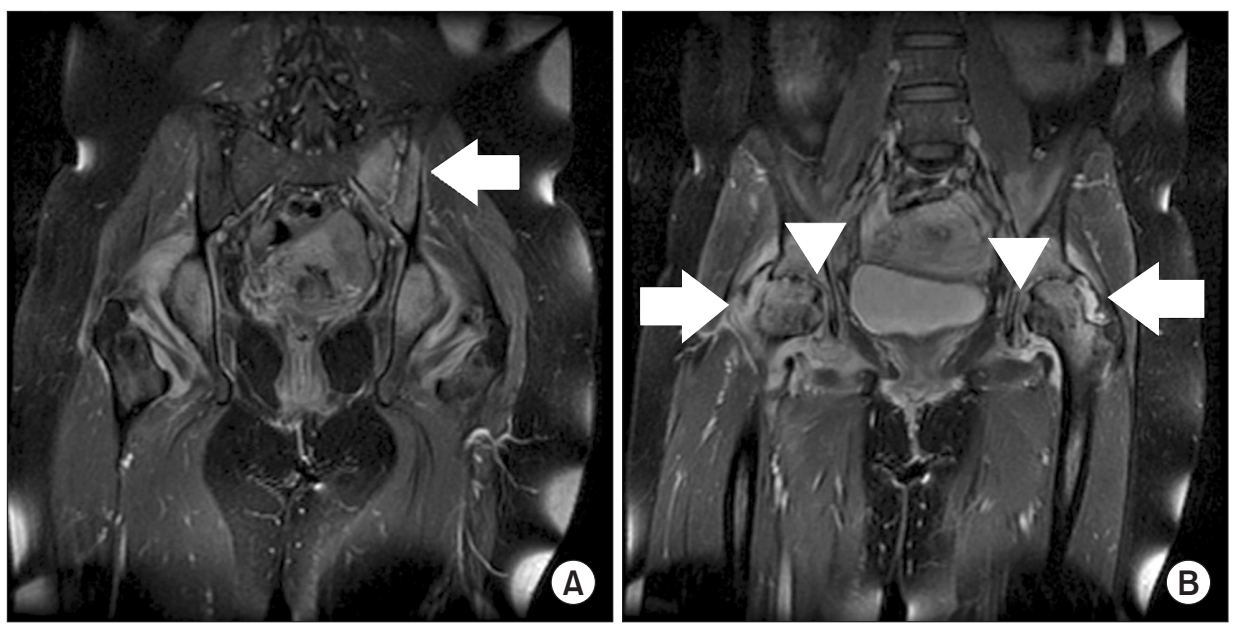

Fig. 2. Magnetic resonance images of bilateral hips at one month after delivery. Coronal T2-weighted image shows a suspicious abscess in the right pectineus muscle (A), bilateral hip joint space narrowing with cortical destruction (arrow head), and surrounding soft tissue edema with enhancement and bilateral hip joint effusion (arrow) (B). 

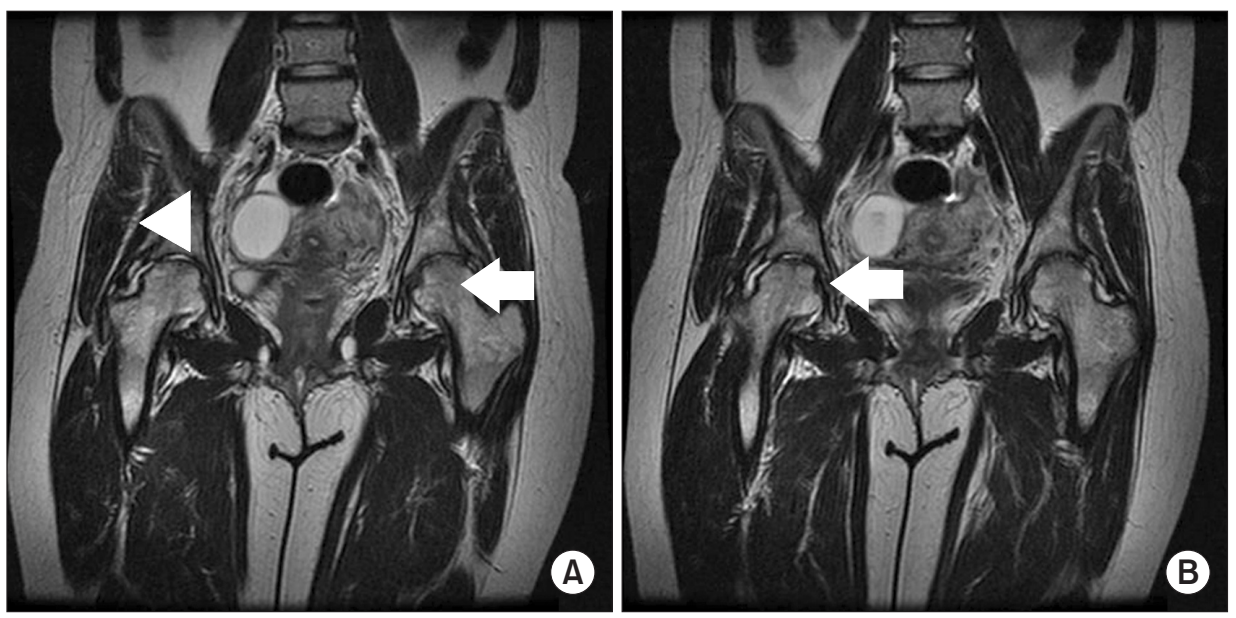

Fig. 3. Magnetic resonance images at four months after delivery. Coronal T2-weighted image demonstrates slightly decreased marrow signal in the left hip (arrow) and a decrease in joint effusion and adjacent soft tissue edema of the right hip (arrow head) (A) but a slight aggravation of the depressed lesion on the anterosuperior aspect of the right femoral head (arrow) (B).

left pyogenic sacroiliitis, and a suspicious abscess in the right pectineus muscle (Fig. 2). We performed emergent ultrasonography (US)-guided aspiration from the pectineus muscle and it proved to be fluid collection and not an abscess. Analysis of joint fluid from the hip joint was also performed, and it demonstrated an increase in white blood cell count, red blood cell count, and lymphocyte count. However, no organism was isolated from the hip joint fluid. Her CRP level was $2.1 \mathrm{mg} / \mathrm{dL}$ and white blood cell count was $2,200 / \mathrm{mm}^{3}$ at the time of transfer.

We stopped the antibiotic therapy and started conservative treatment. After 2 weeks of medical care, her acute symptoms, such as pain and heating sensation were improved and she started receiving rehabilitation treatment. However, limitation of motion (LOM) and pain in bilateral hip joints persisted although rehabilitative therapy was continued for 3 months. MR images of bilateral hip joints were re-obtained and they showed slightly decreased marrow signal in the left hip, sacroiliac joint, and symphysis pubis. They also showed a decrease in joint effusion and adjacent soft tissue edema of the right hip, but a slight aggravation of the depressed lesion on the anterosuperior aspect of the femoral head. Infection with low virulence organism, spondyloarthropathy or postpartum rheumatoid arthritis, was suspected (Fig. 3). We conducted US-guided gun biopsy of synovium in the left hip joint and there was no evidence of inflammation. Her laboratory findings were normal and she had a pain score of 3 on a $0-10$ point visual analogue scale. She was discharged and she continued physical therapy at a local hospital.

Although she continued physical therapy and intermit-

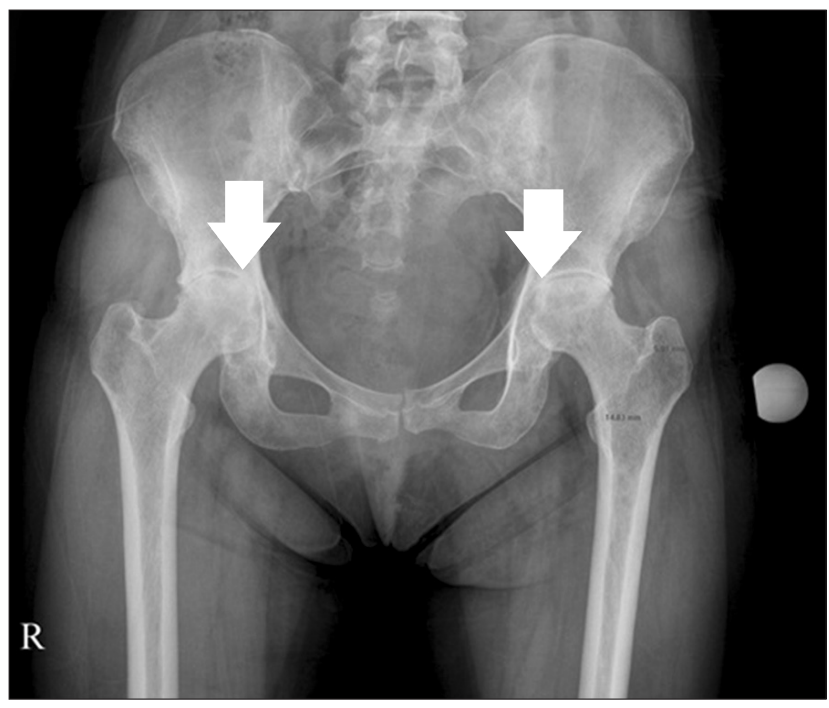

Fig. 4. A radiograph of the hips shows sclerotic changes with flattening of the bilateral femoral heads and joint space narrowing.

tent pain medication for 12 months, LOM of both hip joints increased and it seriously interfered with her daily activities. The range of motion (ROM) of the hip joints was $10^{\circ}$ of extension on the right side and $0^{\circ}$ on the left side. Flexion was also limited to $90^{\circ}$ on the right side and $80^{\circ}$ on the left side. The ROM for abduction and adduction was $30^{\circ}$ and $15^{\circ}$ on the right side, and $25^{\circ}$ and $0^{\circ}$ on the left side. She could barely walk independently and was crutch-bound for most of the time in her daily life. Plain radiography of her hip joints revealed sclerotic changes with flattening of the bilateral femoral heads and joint space narrowing (Fig. 4).

The patient underwent total hip replacement on the left 

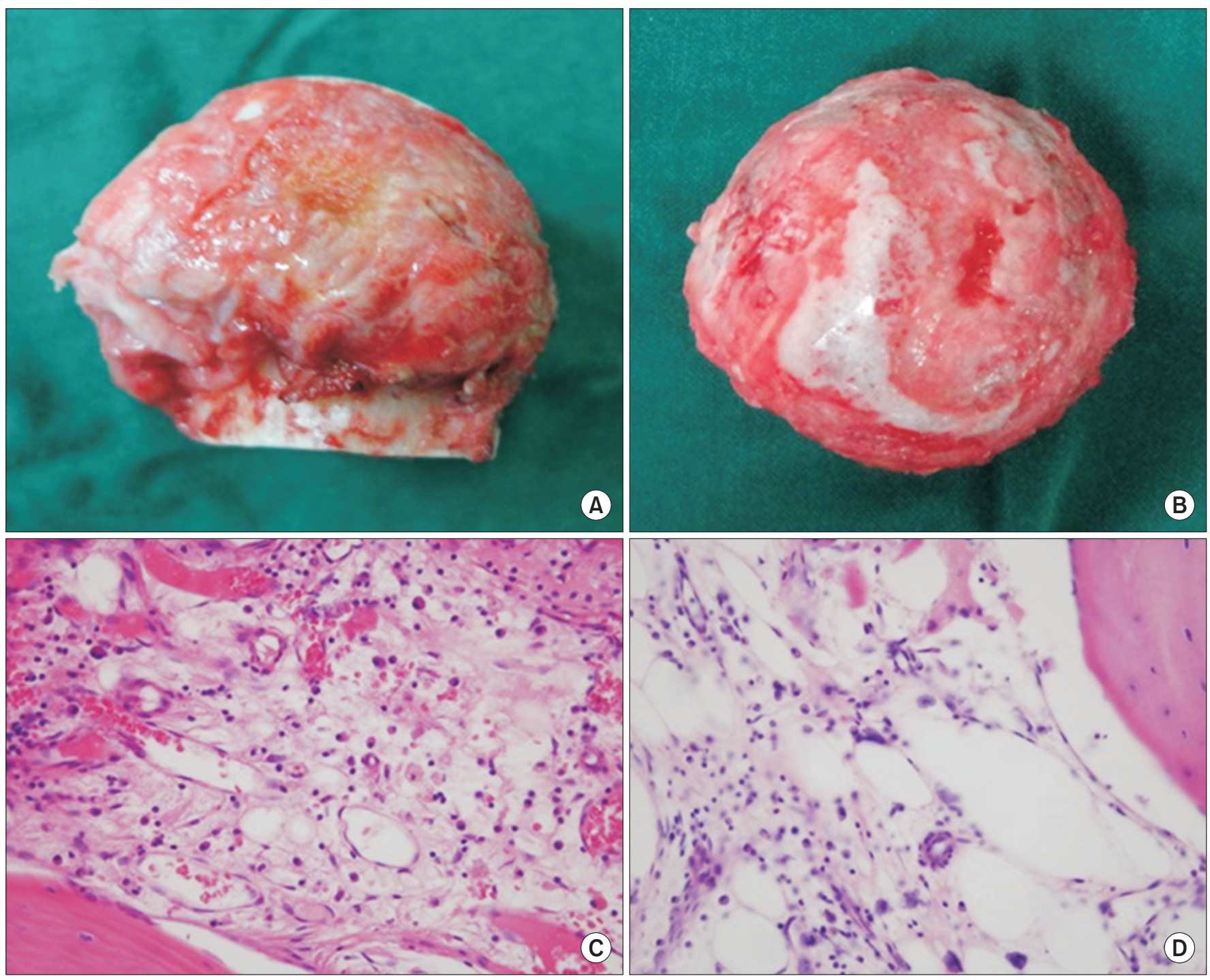

Fig. 5. Gross specimens (A, B) shows a marked erosive lesion of the cartilage surface. Microscopic examination (C, D) revealed mild infiltration of lymphocytes and plasma cells in the medullary space $(\times 400)$.

side because of persistent LOM, and after 2 months, she underwent the same surgery on the right side. Biopsy of the femoral heads demonstrated chronic osteomyelitis with degenerative changes in both sides. Gross specimens showed a marked erosive lesion of the cartilage surface. Microscopic examination revealed mild infiltration of lymphocytes and plasma cells in the medullary space (Fig. 5). After total hip replacement, LOM and pain disappeared in both sides, and she could perform her normal daily activities.

\section{DISCUSSION}

Pregnancy-related pelvic pain is one of the common symptoms during the peripartum period. It is usually caused by change in the center of gravity, secondary to weight gain, pregnancy-related hormonal changes, and joint laxity. The intensity of pain can range from mild to severe. In most cases, the pain disappears soon after delivery and signs of joint infection such as heating sensation, swelling, and tenderness are usually not observed $[2,3]$.

The patient started complaining of pain in the bilateral hip area 12 days after delivery. The pain aggravated gradually, and fever $\left(38.4^{\circ} \mathrm{C}\right)$, leukocytosis $\left(14,250 / \mathrm{mm}^{3}\right)$, and increased CRP level $(15.2 \mathrm{mg} / \mathrm{dL})$ were detected 17 days after delivery. MR image showed osteomyelitis of bilateral hip joints and femoral heads with an abscess in the 
surrounding muscle. The physicians explained the need for joint fluid aspiration and biopsy of the hip joints for proper diagnosis and management. However, the patient and her husband strongly refused the invasive procedure, and the physicians had to initiate empirical antibiotic therapy. Cefminox (2nd generation cephalosporin) was immediately administered to the patient and it was changed to cefmenoxime (3rd generation cephalosporin) one week later because of sustained fever. Although fever subsided after the 5-week antibiotic therapy, the patient developed severe leukocytopenia.

According to previous studies, leukocytopenia is not common but it is one of the well-known complications after cephalosporin therapy [6]. We stopped the antibiotic therapy and leukocytopenia was resolved with conservative treatment.

Pandya et al. [7] reported a case of septic arthritis of the hip joint after delivery. They suggested that peripartum colonization of group B Streptococcus (GBS) caused psoas abscess and subsequent septic hip joint. Although we aspirated and analyzed the hip joint fluid, any specific organism was not isolated from the fluid. We assumed that the etiologic bacteria were not isolated because antibiotic therapy was started before the joint fluid culture [8]. However, we confirmed joint and bone infection from the clinical and radiologic evidence, and laboratory results $[5,9]$. Because many types of bacteria can cause septic joints during pregnancy, accurate bacterial isolation is very important for effective treatment. For example, Pseudomonas aeruginosa, Staphylococcus aureus, Mycoplasma hominis, and GBS are the most commonly isolated organisms during pregnancy [10].

Septic arthritis or osteomyelitis usually develops as a result of hematogenous seeding, direct intrusion, extension from adjacent infection, etc., and the hematogenous spread is the most common cause [5]. The MR images obtained immediately after the onset of fever and infection symptoms of the hip joints demonstrated multiple simultaneous infections, such as osteomyelitis, sacroiliitis, and a suspicious abscess. It seems unlikely that this type of multifocal infection can be caused by direct intrusion or extension from adjacent sites Furthermore, the obstetrician could not identify any injury or open wound that might be a possible source of infection after delivery. In the light of statistical data and multifocal infection sites, we assumed that infection in the patient occurred through hematogenous spread from the episiotomy site or detachment site of the placenta [9]. In this case, GBS or Staphylococcus aureus was the highly suspected etiologic pathogen. Although prophylactic antibiotics (aminoglycoside and 3rd generation cephalosporin) were administered before delivery, the infection could not be prevented and it caused severe destruction of the joints. According to many previous studies, prophylaxis with antibiotics was proved to be beneficial for both the mother and the baby. However, it did not guarantee prevention of all types of infection, which means that we should closely observe the mother after childbirth. Pain in the joints such as the hip or pelvis is a very common symptom during the peripartum period. Many physicians tend to ignore the fact that this type of joint pain could be the symptom of a serious infection or illness. This case highlights the importance of paying attention to minor signs and symptoms that could be related to a serious illness.

The patient continued rehabilitative therapy for 12 months after discharge; however, LOM of the hip joints interfered with her daily activities. Every other conservative treatment failed to restore the range of motion and bilateral total hip replacement had to be performed. After the surgery, the hip joint motion was restored and pain was mostly relieved.

To the best of our knowledge, severe infection such as septic arthritis and osteomyelitis after delivery involving both sides is extremely rare. However, this case proved that hip joint pain after delivery could be due to a destructive joint disease rather than a benign musculoskeletal disorder. Therefore, we should consider early invasive and extensive evaluation with high suspicion even when the patients present with only minor or common symptoms.

\section{CONFLICT OF INTEREST}

No potential conflict of interest relevant to this article was reported.

\section{REFERENCES}

1. Bastiaenen CH, Bastiaanssen JM, de Bie RA. Comment on Gutke A, Ostgaard HC, Oberg B. Pelvic girdle pain and lumbar pain in pregnancy: a cohort study of the consequences in terms of health and function- 
ing. Spine 2006;31:E149-55. Spine (Phila Pa 1976) 2006;31:2406.

2. Damen L, Buyruk HM, Guler-Uysal F, Lotgering FK, Snijders CJ, Stam HJ. Pelvic pain during pregnancy is associated with asymmetric laxity of the sacroiliac joints. Acta Obstet Gynecol Scand 2001;80:1019-24.

3. Ostgaard HC, Andersson GB. Postpartum low-back pain. Spine (Phila Pa 1976) 1992;17:53-5.

4. Gamble K, Dardarian TS, Finstein J, Fox E, Sehdev H, Randall TC. Osteomyelitis of the pubic symphysis in pregnancy. Obstet Gynecol 2006;107(2 Pt 2):477-81.

5. Shirtliff ME, Mader JT. Acute septic arthritis. Clin Microbiol Rev 2002;15:527-44.

6. Whitman CB, Joseph JM, Sjoholm LO. Cephalosporininduced leukopenia following rechallenge with cefoxitin. Ann Pharmacother 2008;42:1327-32.

7. Pandya N, Accardi K, Israelite C. Septic arthritis of the hip following group B Streptococcal psoas abscess in a postpartum patient resulting in total hip arthroplasty. Internet J Orthop Surg [Internet]. 2007 [cited 2015 May 20];6. Available from: https://ispub.com/ IJOS/6/2/11638.

8. van der Heijden IM, Wilbrink B, Vije AE, Schouls LM, Breedveld FC, Tak PP. Detection of bacterial DNA in serial synovial samples obtained during antibiotic treatment from patients with septic arthritis. Arthritis Rheum 1999;42:2198-203.

9. Bonhoeffer J, Haeberle B, Schaad UB, Heininger U. Diagnosis of acute haematogenous osteomyelitis and septic arthritis: 20 years experience at the University Children's Hospital Basel. Swiss Med Wkly 2001;131:575-81.

10. Howell JD, Sheddon RJ. Septic arthritis of the hip complicating pregnancy. Postgrad Med J 1995;71:3167. 